\title{
Supporting information for \\ Kinetic and thermodynamic driving factors in the assembly of phenylalanine-based modules
}

Dor Zaguri ${ }^{1,+}$, Manuela R. Zimmermann ${ }^{2,+}$, Georg Meisl ${ }^{2}$, Aviad Levin ${ }^{2}$, Sigal Rencus-Lazar ${ }^{1}$, Tuomas P. J. Knowles ${ }^{2,3, *}$, and Ehud Gazit ${ }^{1,4, *}$

1 The Shmunis School of Biomedicine and Cancer Research, Tel Aviv University, Tel Aviv 6997801, Israel.

${ }^{2}$ Yusuf Hamied Department of Chemistry, University of Cambridge, Cambridge, CB2 1EW, United Kingdom.

${ }^{3}$ Cavendish Laboratory, University of Cambridge, Cambridge, CB3 OHE, United Kingdom.

${ }^{4}$ BLAVATNIK CENTER for Drug Discovery for Drug Discovery, Tel Aviv University, Tel Aviv 6997801, Israel.

† These authors contributed equally: D. Zaguri and M.R. Zimmermann.

*To whom correspondence should be addressed: tpjk2@cam.ac.uk and ehudga@tauex.tau.ac.il.

\section{Derivation of equation (3) in the main text}

Classical nucleation theory (CNT) is a frequently applied model to study nucleation kinetics. It is based on the idea of balancing the bulk energy gained from forming a new phase with the energy penalty of forming a new phase. The main underlying assumptions are local equilibrium conditions, negligible finite size effects of the nucleus, and a one-step aggregation mechanism. As stated in equation (2) in the main text, CNT describes the free energy of a spherical cluster comprising $n$ monomers by

$$
\Delta G(n)=\underbrace{-n k_{B} T \ln S}_{\text {bulk stabilisation }}+\underbrace{\left(36 \pi v^{2}\right)^{1 / 3} \gamma n^{2 / 3}}_{\text {surface destabilisation }}
$$

where $k_{B}$ is the Boltzmann constant, $T$ the absolute temperature, $S=c / c_{s}$ the supersaturation, $\gamma$ the effective surface tension and $v$ the partial volume of a monomer in the condensed phase. The nucleus corresponds to the least stable cluster and thus to the critical point of $\Delta G(n)$. Its size is given by

$$
N^{*}=\frac{32 \pi \gamma^{3} v^{2}}{3 k_{B}^{3} T^{3}(\ln S)^{3}}
$$

The nucleus size indicates the number of monomers actively participating in the rate-limiting step of nucleation. It is important to note that this amount was found to generally be a lower boundary for the number of monomers in the physical nucleus because not all monomers in this critical cluster might be actively involved in the rate limiting step. ${ }^{1}$ 
The size of the nucleus, equation (S2), sets the free energy barrier of nucleation through equation (2) in the main text,

$$
\Delta G^{*}=\Delta G\left(n=N^{*}\right)=\frac{16 \pi v^{2} \gamma^{3}}{3 k_{B}^{3} T^{2} \ln ^{2} S} .
$$

Assuming Arrhenius-type kinetics, the rate $J$ of nucleation is

$$
J=A_{J} \exp \left(-\frac{\Delta G^{*}}{k_{B} T}\right)=A_{J} \exp \left(-\frac{16 \pi v^{2} \gamma^{3}}{3 k_{B}^{3} T^{3} \ln ^{2} S}\right)
$$

where $A_{J}$ is a constant, often referred to as kinetic pre-factor. $J$ is inversely proportional to the lag time, $\tau$. After simple algebra, we obtain equation (3) in the main text

$$
\ln \tau=\frac{16 \pi v^{2} \gamma^{3}}{3 k_{B}^{3} T^{3} \ln ^{2} S}+A
$$

for which $A$ summarises (the natural logarithm of) the proportionality constants. 
a

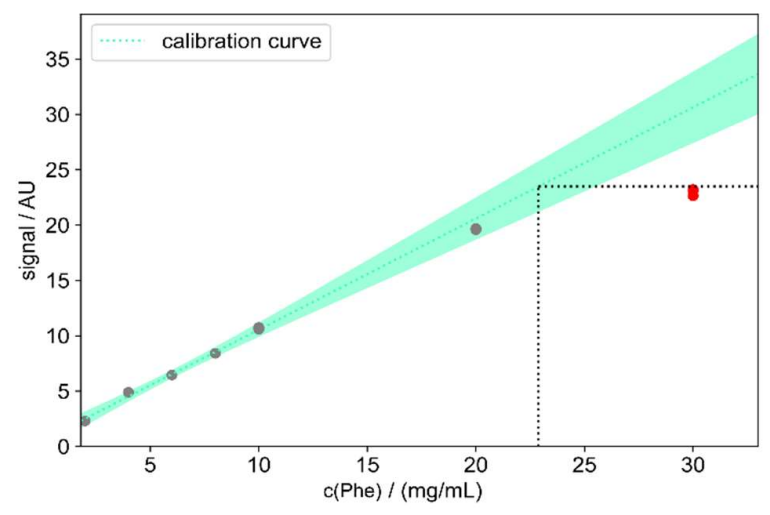

b

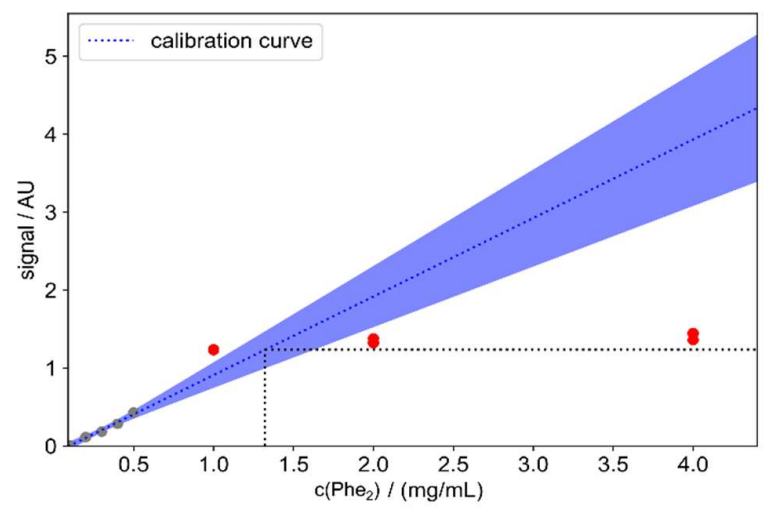

C

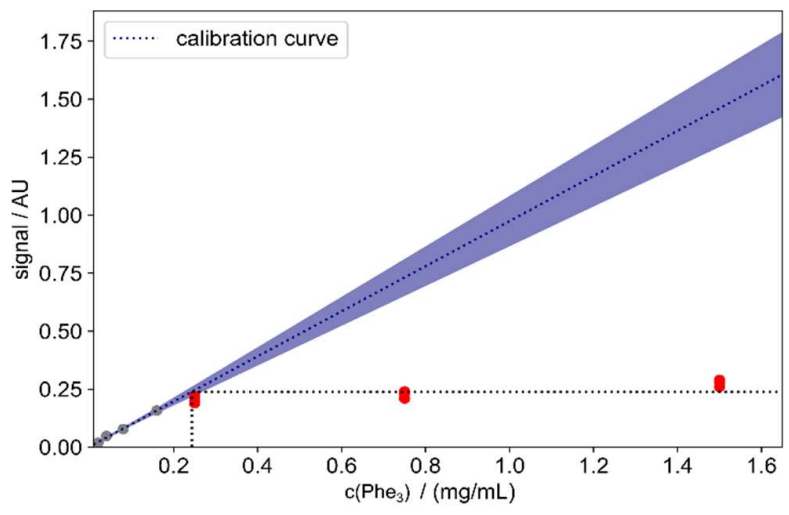

Figure S1: Solubility measurements: The solubility was determined as the average constant monomer concentration in the supernatant in an aggregated solution (red points and dark dotted lines). A calibration curve (coloured dotted line) was used to relate the absorbance signal to a monomer concentration. (a) Phe. (b) $\mathrm{Phe}_{2}$. (c) $\mathrm{Phe}_{3}$. 
Table S1: Values of solubility and effective surface tension according to CNT for the different tested systems. Errors indicate one standard deviation.

\begin{tabular}{lccc}
\hline & Phe & Phe $_{2}$ & Phe $_{3}$ \\
\hline Solubility $c_{s} /(\mathrm{g} / \mathrm{L})$ & $23 \pm 1$ & $1.3 \pm 0.2$ & $0.24 \pm 0.1$ \\
Effective surface tension $\gamma /\left(\mathrm{mJ} / \mathrm{m}^{2}\right)$ & $4.3 \pm 0.1$ & $3.9 \pm 0.2$ & $3.0 \pm 0.5$ \\
\hline
\end{tabular}

\section{References}

(1) Šarić, A.; Michaels, T. C. T.; Zaccone, A.; Knowles, T. P. J.; Frenkel, D. Kinetics of Spontaneous Filament Nucleation via Oligomers: Insights from Theory and Simulation. J. Chem. Phys. 2016, 145 (21). https://doi.org/10.1063/1.4965040. 